\title{
Oxidative Stress in IgA Nephropathy
}

\author{
R. Coppo R. Camilla A. Amore L. Peruzzi \\ Nephrology, Dialysis, Transplantation, Regina Margherita University Hospital, Turin, Italy
}

\section{Key Words}

Advanced oxidation protein products $\cdot$ Chronic kidney disease $\cdot \lg$ A nephropathy $\cdot$ Oxidative stress $\cdot$ Pathogenesis of IgAN

\begin{abstract}
IgA nephropathy (IgAN) is characterized by mesangial deposits of IgA1, likely due to accumulation of IgA immune complexes. The activation of intracellular signaling mostly results in oxidative stress, as detected in mesangial cells cultured with aberrantly glycosylated IgA or IgA aggregates and in renal biopsies of patients with IgAN. Signs of altered oxidation/antioxidation balance have been detected in sera and/or in erythrocytes of patients with IgAN, including increased levels of lipoperoxide or malondialdehyde and reduced activity of superoxide dismutase, catalase and glutathione peroxidase. Moreover, increased levels of a marker of oxidative stress, advanced oxidation protein products (AOPPs), have been reported to be significantly associated with proteinuria and disease progression in patients with IgAN. AOPPs are often carried by albumin and can in turn enhance the oxidative stress in the circulation. Recent research suggests that the nephrotoxicity of aberrantly glycosylated IgA1 in IgAN is enhanced in the presence of systemic signs of oxidative stress, and it is tempting to hypothesize that the level of the oxidative milieu conditions the different expression and progression of IgAN.
\end{abstract}

Copyright $\odot 2010$ S. Karger AG, Basel
(C) 2010 S. Karger AG, Basel

$1660-2110 / 10 / 1163-0196 \$ 26.00 / 0$

Fax +4161306 1234 E-Mail karger@karger.ch www.karger.com
Accessible online at:

www.karger.com/nec

\section{Overview of the Present Hypothesis on the Pathogenesis of IgA Nephropathy}

The hallmark of IgA nephropathy (IgAN) is mesangial deposits of mostly polymeric IgA1, likely due to an accumulation of IgA immune complexes [1]. IgAN is characterized by a dysregulation of the immune system, with an altered response to environmental antigens eventually leading to circulation and mesangial deposition of aberrantly glycosylated polymeric IgA1 [2]. IgA1 is heavily glycosylated, with O-glycosylated carbohydrate chains based on $\mathrm{N}$-acetylgalactosamine (GalNAc) residues, usually extended with galactose (Gal) to form Gal $11,3 \mathrm{GalNAc}$, which may be covered with $\alpha 2,6$ - and/or $\alpha 2,3$-sialic acid (Neu5Ac or Sia) [2]. Aberrantly glycosylated IgA1 synthesis can be triggered by exposure to pathogens of viral or bacterial origin. The alteration in galactosylation and sialylation of the glycans of IgA1 modifies the three-dimensional structure of the molecule and affects its affinity and binding to intrinsic mesangial glycoproteins (e.g. fibronectin, laminin, collagens) and plasma proteins, including mannose-binding lectin and properdin, also favoring self-aggregation and IgG autoantibody formation. Moreover, aberrantly glycosylated IgA1 shows increased reactivity with mesangial cells, perhaps by interaction with IgA receptors (such as the transferrin receptor, CD71) located on the surface of mesangial cells.

\section{Oxidants in Chronic Kidney Disease}

It is known that patients with uremia present with an increase in plasma protein oxidation, including thiol residue oxidation and carbonyl formation together with a de-

Rosanna Coppo, MD

Regina Margherita University Hospital

Piazza Polonia 94

IT-10126 Turin (Italy)

Tel. +39011313 5848, E-Mail rosanna.coppo@unito.it 
crease in important intracellular and circulating antioxidants, of which thiol groups of albumin are quantitatively the most important [3]. Besides uremia, experimental evidence supports the role of oxidants not only in inducing tubulointerstitial damage which accompanies progression, but also in the early stages of glomerular damage [4]. Enhanced generation of oxidants by infiltrating leukocytes has been demonstrated in anti-Thyl.1 and antiGBM-induced glomerulonephritis, and plays a major role in inducing exudative and proliferative glomerular changes. Leukocytes are thought to cause proteinuria by degrading the glomerular basement membrane activating a latent metalloproteinase by $\mathrm{HOCl}$ or a similar oxidant generated by the myeloperoxidase- $\mathrm{H}_{2} \mathrm{O}_{2}$-halide system.

Proteinuric experimental models lacking infiltrating leukocytes, like nephrosis induced by injection of puromycin aminonucleoside, have also demonstrated a role of oxidants. The role of oxidative stress in inducing proteinuria is supported by a recent report which detects a causative link between advanced oxidation protein products (AOPPs) and podocyte depletion [5]. AOPPs are a family of oxidized, dityrosine-containing protein-insoluble products, resistant to proteolysis, generated by oxidative stress associated with monocyte activation, myeloperoxidase release, and production of reactive oxygen species. Increased levels of AOPPs are associated with high proteinuria, monocyte interstitial infiltration and glomerulosclerosis in murine models of remnant kidneys. AOPPs activate the intrarenal redox-sensitive pathway with nuclear translocation of nuclear factor $\kappa \mathrm{B}(\mathrm{NF}-\kappa \mathrm{B})$ and synthesis of pro-inflammatory and pro-sclerotic mediators [6]. AOPPs induce podocyte apoptosis when injected in experimental animals and when incubated in podocyte cultures at remarkably low doses. AOPPs induce intracellular superoxide generation in podocytes by a mechanism involving nicotinamide adenine dinucleotide phosphate (NADPH) oxidase. The cascade of intracellular signaling events finally leads to p53-dependent apoptosis [5]. The conclusion from this recent report suggests that AOPP-mediated apoptosis plays a critical role in promoting proteinuria and accelerating glomerular sclerosis.

\section{Activation of the Oxidative Stress Pathway in IgAN}

The activation of intracellular signaling mostly results in oxidative stress, as detected in mesangial cells cultured with aberrantly glycosylated IgA or IgA aggregates $[7,8]$ and in renal biopsies of patients with IgAN, similar to what has been detected in diabetic nephropathy [9]. Signs

Oxidative Stress in IgA Nephropathy of altered oxidation/antioxidation balance have been detected in sera and/or in erythrocytes of patients with IgAN, including increased levels of lipoperoxide or malondialdehyde and reduced activity of superoxide dismutase, catalase and glutathione peroxidase $[10,11]$. Moreover, increased levels of AOPPs have been recently reported to be significantly associated with proteinuria and disease progression in patients with IgAN [12]. AOPPs are often carried by albumin and can in turn enhance the oxidative stress in the circulation. AOPPs are detected in patients with advanced chronic renal failure, but in IgAN they are independent early predictors of poor renal outcome [12].

\section{Variable Natural History of IgAN}

One of the most fascinating and still unanswered questions concerning IgAN is the extreme variability of natural history in individual patients, ranging from rapidly progressive cases to 'lanthanic' IgA innocent deposits (4-16\%; averaging about 70,000 per million population) [13]. It can be debated whether these are 'innocent' findings or whether these subjects represent a state of 'pre-disease' awaiting another event (a second 'hit') to bring the disease to full clinical and recognizable expression. Moreover, the disease can show no sign of progression at all even after decades in $5-50 \%$ of patients, or progress to end-stage renal failure within 20 years in $80 \%$ of patients.

\section{New Working Hypothesis on Pathogenesis of Progressive IgAN}

The unique role of circulating aberrantly $\operatorname{IgA} 1$ in inducing IgAN renal lesions and the wide spectrum of disease progression has been questioned, as similar Ig A $1 \mathrm{ab}-$ normalities have been described in both familial and sporadic cases of IgAN as well as in healthy first-degree relatives but not in their spouses. In patients with IgAN, individual O-galactosylation patterns of serum IgA1 was reported by Smith and Feehally's group [13] to remain constant over long periods of time. These were also loosely associated with severity of renal disease, but the overlap between different groups of progressors or non-progressors suggested that altered IgA1 O-glycosylation alone is insufficient to trigger progression.

In a collaborative study among Italian and US researchers, presented at the last IgA Nephropathy Net- 
work Meeting in 2009 [13], we showed the concomitant presence in patient sera of aberrantly glycosylated IgA1 and signs of oxidative stress, increased AOPP and decreased free thiol groups of serum albumin. Total levels of aberrantly glycosylated IgA1 resulted in being only weakly associated with clinical activity and progression of renal damage. However, the relative degree of galactose deficiency of IgA1 in association with the concomitant activation of the oxidation pathway resulted in being correlated with proteinuria and informative for disease progression. These data suggest that the nephrotoxicity of aberrantly glycosylated IgA1 in IgAN is enhanced in the presence of systemic signs of oxidative stress.

It is of interest that the increase in AOPP levels in patients with IgAN were reported to be correlated with levels of proteinuria [12] and were detected in patients with preserved renal function, hence suggesting an early activation of the process in IgAN, largely before reaching uremia. It may be speculated that in patients with IgAN, mesangial cells are under the effect not only of deposited aberrantly glycosylated IgA1 but also of circulating and locally generated AOPPs. Both mediators trigger the oxidative stress pathways, notably NF- $\kappa$ B nuclear translocation favoring inflammation and sclerosis $[6,7]$. Recently, we also reported that peripheral lymphomonocytes from patients with IgAN have an increased nuclear translocation of this powerful transcription factor, which can support a condition of systemic activation of oxidation [14].

The role of the oxidative milieu as a risk factor for progression of IgAN as well as for mortality has recently also been supported by the association with the polymorphism in the promoter region of the heme oxygenase-1 conditioning the low activity of this system [15]. The support for this hypothesis would be that antioxidant therapy is effective in treating IgAN. The rationale of some therapeutic approaches to IgAN, like fish-oil/omega-3 polyunsaturated fatty acids as well as vitamin E, was based on the aim of downregulating the oxidative status, however, results were not convincing. Like other unfruitful attempts to modulate the oxidoreductive balance in human diseases, it is still debated whether the inconsistent clinical results were affected by inadequate effectiveness of the agents used or by escape mechanisms. However, recent studies indicate that the area is still open to new strategies focusing on the contribution to renal damage induced by oxidative stress pathway activation in IgAN. Genetic control of antioxidant responses may be crucial in individual patients, and studies investigating regulation/augmentation of antioxidant responses seem to be a very promising field of future research.

\section{References}

1 D'Amico G: Natural history of idiopathic IgA nephropathy and factors predictive of disease outcome. Semin Nephrol 2004;24: 179-196.

-2 Coppo R, Amore A: Aberrant glycosylation in IgA nephropathy (IgAN). Kidney Int 2004;65:1544-1547.

$\checkmark 3$ Himmelfarb J, McMonagle E: Albumin is the major plasma protein target of oxidant stress in uremia. Kidney Int 2001;60:358-363.

$\checkmark 4$ Shah SV, Baliga R, Rajapurkar M, Fonseca VA: Oxidants in chronic kidney disease. J Am Soc Nephrol 2007; 18:16-28.

$\checkmark 5$ Liu Y: Advanced oxidation protein products: a causative link between oxidative stress and podocyte depletion. Kidney Int 2009;76: 1125-1127.

6 Guo ZJ, Niu HX, Hou FF, Zhang L, Fu N, Nagai R, Lu X, Chen BH, Shan YX, Tian JW, Nagaraj RH, Xie D, Zhang X: Advanced oxidation protein products activate vascular endothelial cells via a RAGE-mediated signalling pathway. Antioxid Redox Signal 2008; 10:1699-1712.
7 Gómez-Guerrero C, González E, Hernando P, Ruiz-Ortega M, Egido J: Interaction of mesangial cells with IgA and IgG immune complexes: a possible mechanism of glomerular injury in IgA nephropathy. Contrib Nephrol. Basel, Karger, 1993, vol 104, pp 127-137.

8 Chen HC, Guh JY, Chang JM, Lai YH: Differential effects of circulating IgA isolated from patients with IgA nephropathy on superoxide and fibronectin production of mesangial cells. Nephron 2001;88:211-217.

$\checkmark 9$ Suzuki D, Miyata T, Saotome N, Horie K, Inagi R, Yasuda Y, Uchida K, Izuhara Y, Yagame M, Sakai H, Kurokawa K: Immunohistochemical evidence for an increased oxidative stress and carbonyl modification of proteins in diabetic glomerular lesions. J Am Soc Nephrol 1999; 10:822-32

10 Vas T, Wagner Z, Jenei V, Varga Z, Kovacs T, Wittmann I, Schinzel R, Balla J, Heidland A, Nagy J: Oxidative stress and non-enzymatic glycation in IgA nephropathy Clin Nephrol 2005;64:343-351.

$\checkmark 11$ Chen JX, Zhou JF, Shen HC: Oxidative stress and damage induced by abnormal free radical reactions and IgA nephropathy. J Zhejiang Univ Sci B 2005;6:61-68.
12 Descamps-Latscha B, Witko-Sarsat V, Nguyen-Khoa T, Nguyen AT, Gausson V, Mothu N, Cardoso C, Noël LH, Guérin AP, London GM, Jungers P: Early prediction of $\operatorname{IgA}$ nephropathy progression: proteinuria and AOPP are strong prognostic markers. Kidney Int 2004;66:1606-1612.

13 Coppo R, Feehally J, Glassock RJ: IgA nephropathy at two score and one. Kidney Int 2010;77:181-186.

- 14 Coppo R, Camilla R, Alfarano A, Balegno S, Mancuso D, Peruzzi L, Amore A, Dal Canton A, Sepe V, Tovo P: Upregulation of the immunoproteasome in peripheral blood mononuclear cells of patients with IgA nephropathy. Kidney Int 2009;75:536-541.

$\checkmark 15$ Chin HJ, Cho HJ, Lee TW, Na KY, Yoon HJ, Chae DW, Kim S, Jeon US, Do JY, Park JW, Yoon KW, Shin YT, Lee KW, Na KR, Cha DR, Kang YS: Progressive REnal disease and Medical Informatics and gEnomics Research (PREMIER) members. J Korean Med Sci 2009;24(suppl):S30-S37. 


\section{Editorial Comment}

M. El Nahas, Sheffield

The review by Coppo et al. revisits the pathogenesis of IgA nephropathy, one of the most common causes of glomerulonephritis worldwide. It challenges the hypothesis that abnormal IgA1 O-glycosylation is sufficient on its own to explain the progression of the disease. It suggests very elegantly that in addition to abnormalities of $\operatorname{IgA} 1$, oxidant stress (generation of advanced oxidation protein products - AOPPs) is necessary to promote the progression of glomerular damage and CKD. Of interest, a recent report of IgAN after bone marrow transplantation suggests that the pathogenesis of the disease is due to a multihit process associated with glomerular deposition of galactose-deficient IgA1. In this report from Boston, two bone marrow transplant recipients provide a unique insight into the kinetics of overproduction of galactosedeficient IgA1 and its glomerular deposition in IgAN [1]. These cases may indirectly lend support to the Coppo hypothesis as her group has recently shown that circulating lymphomonocytes from patients with IgAN have an increased nuclear translocation of NF- $\kappa B$, a powerful pro-inflammatory transcription factor, which would promote systemic activation of oxidation in IgAN [2]. The story of IgAN may be unravelling as a multi-hit condition with abnormalities of O-glycosylation of IgA1, pro-inflammatory cells as well as generation of pro-inflammatory and pro-sclerotic AOPPs.

\section{References}

1 Hu SL, Colvin GA, Rifai A, Suzuki H, Novak J, Esparza A, Farooqi S, Julian BA: Glomerulonephritis after hematopoietic cell transplantation: IgA nephropathy with increased excretion of galactose-deficient IgA1. Nephrol Dial Transplant 2010, in press.

- Coppo R, Camilla R, Alfarano A, Balegno S, Mancuso D, Peruzzi L, Amore A, Dal Canton A, Sepe V, Tovo P: Upregulation of the immunoproteasome in peripheral blood mononuclear cells of patients with IgA nephropathy. Kidney Int 2009;75:536-541. 